\title{
TRIGEMINAL NEURALGIA: UPDATED MANAGEMENT
}

\author{
Jacques Magnan'1, Bhavin Parikh², Pradeep Vundavalli ${ }^{3}$ \\ ${ }^{1}$ University of Aix-Marseille, France \\ ${ }^{2}$ Bharti ENT Care Hospital, Vadodora, India \\ ${ }^{3}$ Asian ENT Hospital, Vishakhapatnam, India
}

\begin{abstract}
The so-called idiopathic trigeminal neuralgia is easily diagnosed by the clinical history of the patient and present a constant cause, a neurovascular conflict in the cerebellopontine angle in the majority of cases, which is clearly identified by MRI assessment. Knowing the cause, after a trial period with a medical treatment, a curative surgical solution can be now offered to the patient when the medications do not provide a pain-free result or when the patients wish a more definitive solution. Combining the use of both operating microscope and endoscope increases the efficiency of the neurovascular decompression and decreases drastically the potential morbidity of the surgical procedure. From 355 patients suffering of trigeminal neuralgia who underwent endoscope-assisted retrosigmoid approach, the authors present their experiences and results.
\end{abstract}

Keywords: trigeminal neuralgia

\section{INTRODUCTION}

The classical trigeminal neuralgia (TN) is a monotonously repetitive severe shooting pain in the sensitive distribution of the trigeminal nerve $(\mathrm{V})$, easily diagnosed by the history obtained from the patient. It was also called essential or idiopathic neuralgia, because the cause has long been misunderstood. Currently, there is a considerable evidence that in the most of the cases cranial nerve $(\mathrm{CN}) \mathrm{V}$ is compressed by a vascular structure in the cerebellopontine angle (CPA). The classical typical essential or idiopathic trigeminal neuralgia can "be tied into a single etiolog-

Address for correspondence:

Jacques Magnan

University of Aix-Marseille

58 Charles Livon Blvd

13007 Marseille

France

e-mail:jypmagnan@gmail.com

Received: May 9, 2021

Accepted: June 16, 2021 ic package" (1) of "neurovascular compression syndrome". Knowing the cause, a curative solution can finally be proposed to the patient.

The medical treatment with anticonvulsant drugs has been developed primarily as a consequence of Trousseau's hypothesis: tic douloureux or epileptiform neuralgia (2), that the attacks of pain suffered by the patients are due to paroxysmal activity in the trigeminal system similar to the cerebral paroxysmal discharges in epilepsy (3). However, the etiology and pathogenesis of trigeminal neuralgia has remained a subject of controversy.

Dandy (4), Gardner (5), and Jannetta (6) were the pioneers to promote the peripheral concept of neurovascular compression or cross-compression syndrome and to perform surgical decompression with the resolution of the patient's symptoms. After a long period of little acceptance, the neurovascular decompression procedure has been popularized by several technological improvements: MRI assessment in T2 sequences, minimally invasive techniques with endoscope-assisted microsurgery $(7,8)$. 


\section{DIAGNOSIS}

\section{Clinical Features}

Trigeminal neuralgia is a disease of mid-life. Three out of four times the pain is felt after 50 years, with an average of 55 years and range of 18 to 83 years.

The pain of TN is paroxysmal, sharp, often compared to electric shock, brief episodes of pain with periods of remission in between but the patient has anxiety for the next episode. The pain commonly strikes the third (mandibular) division of the nerve along the jaw and the lower teeth or tongue and less commonly affects the second (maxillary) and first (ophthalmic) division. The pain is provoked by speech, chewing, brushing teeth, wind or by the light tactile of the "trigger zone" (unilateral cheek and/or nose). The phenomenon disappears at night, during sleep. Trigeminal neuralgia usually begins as a relapsing disease with pain-free intervals, which grow shorter. There is no sensory deficit, no decrease in corneal reflex, and no symptom in the other cranial nerve territories.

It is essential to distinguish typical TN from the atypical one, secondary or symptomatic TN with a constant background burning or aching pain or with sensory loss or without trigger zone. Generally, the underlying cause is not a neurovascular conflict and does not respond to surgical decompression.

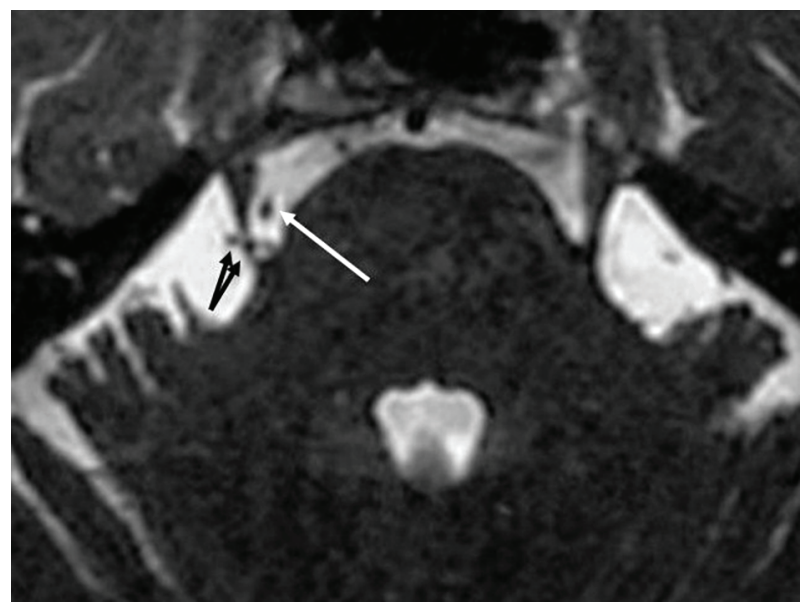

\section{MRI Assessment}

MRI is an essential element in the work-up of these pathologies. MRI is able to reproduce the anatomy of the CPA and to demonstrate the presence of a neurovascular conflict $(9,10)$. It also eliminates other potential causes such as a tumor process or multiple sclerosis. MRI establishes the diagnosis, accurately assesses the responsible vessels, and finally dictates the indication for surgery (9).

MRI in both T1 and T2 three-dimensional Fourier transform (3DFT) sequences is the most effective method of delineating both the cranial nerves and the surrounding vascular structures in the CPA. The protocol for imaging that we follow includes beginning with whole brain T2 or FLAIR sequence to exclude multiple sclerosis, followed by axial \& coronal $\mathrm{Tl}$ (preferably with contrast to look for venous cause) imaging of the brainstem and CPA cistern to look for cranial neuritis, perineural and cisternal tumors. High-resolution thin-section T2 imaging of the brainstem and CPA cistern is performed next and is the best sequence to look for an offending artery. The T2 is carried out using constructive interference in steady state (3D CISS) sequences offering high spatial resolution and excellent contrast resolution and depict both the artery and the vein responsible for the neurovascular conflict. Imaging of the CPA is done with serial thin slices, preferably of 0.4 mm thickness.

To confirm the diagnosis, the single presence of a neurovascular contact is not sufficient. Several ra-

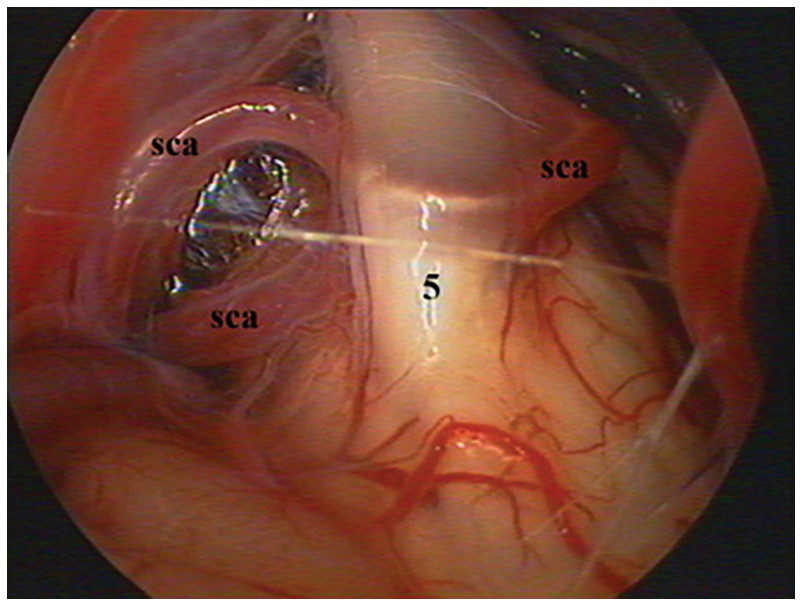

Fig. 1. A. MRI, axial T2 CISS sequence showing a perpendicular contact between the SCA (white arrow) and its branches (black arrows) with the trigeminal nerve at its cistern course, and a reduction in diameter of the trigeminal nerve compared to the opposite side. B. Endoscopic view in same patient of the distorted trigeminal nerve (5) by the SCA. 


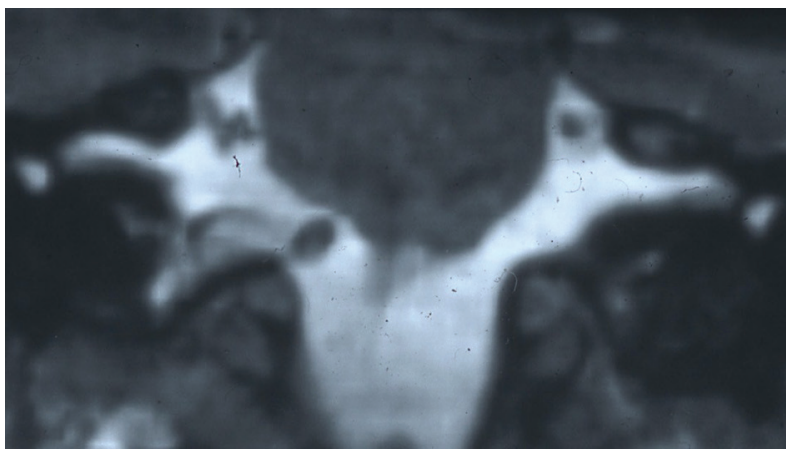

Fig. 2. MRI coronal plane, T2 CISS sequence showing cross-compression of the right trigeminal nerve

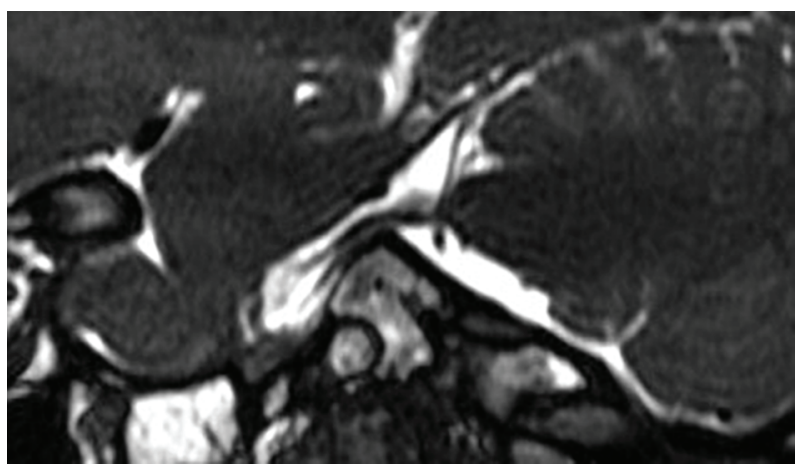

Fig. 3. MRI sagittal plane, T2 CISS sequence showing the trigeminal nerve from the Meckel cave to the pons crossed by the SCA as shown in the endoscopic picture of the right cerebellopontine angle (CPA) with both caudal and rostral branches of SCA impinging into $V$.

diological criteria are required including a perpendicular contact between the vascular loop and the trigeminal nerve at its root entry zone (REZ), visualized along two different perpendicular planes, and a distortion of the nerve or other neural structures.
For the trigeminal nerve, the reduction in diameter is an additional criterion. The most common offending vessel is the superior cerebellar artery (SCA) (Fig. 1A and $1 \mathrm{~B})$, but in around $20 \%$ of cases, a venous structure (aberrant trigeminal vein) might be the cause of the conflict, either alone or in combination with an artery, and it is visible only on T1 with gadolinium. The axial plane offers the most comprehensive and demonstrative images, coronal and sagittal planes confirm and precisely predict the course of the offending artery (Fig. 2, Fig. 3).

The offending vessels are, in order of frequency, SCA (79\%), an aberrant trigeminal vein (29\%), then the ectasia of the vertebrobasilar artery (Fig. 4. A, B, C) and anterior inferior cerebellar artery (AICA) in $5 \%$. The exact site of the conflict on the trigeminal nerve is at root entry zone (REZ) in 33\% of cases, at the cisternal course of the nerve in $50 \%$ of the cases and at the entrance of the nerve in Meckel's cave in $17 \%$ of cases (9).

\section{THERAPEUTIC MANAGEMENT FOR TRIGEMINAL NEURALGIA}

\section{First Line: Medical}

Typical TN usually responds well to medical treatment trial with carbamazepine, at least initially and is pathognomonic of diagnosis. Carbamazepine is started as a first-line drug in doses of $100 \mathrm{mg}$ bid and increased as per requirement up to 800-1000 mg per day based on the tolerance of the patient. Even though carbamazepine has stronger evidence (11), oxcarbazepine in the doses of 600-1800 mg per day is generally considered to be better tolerated (12).
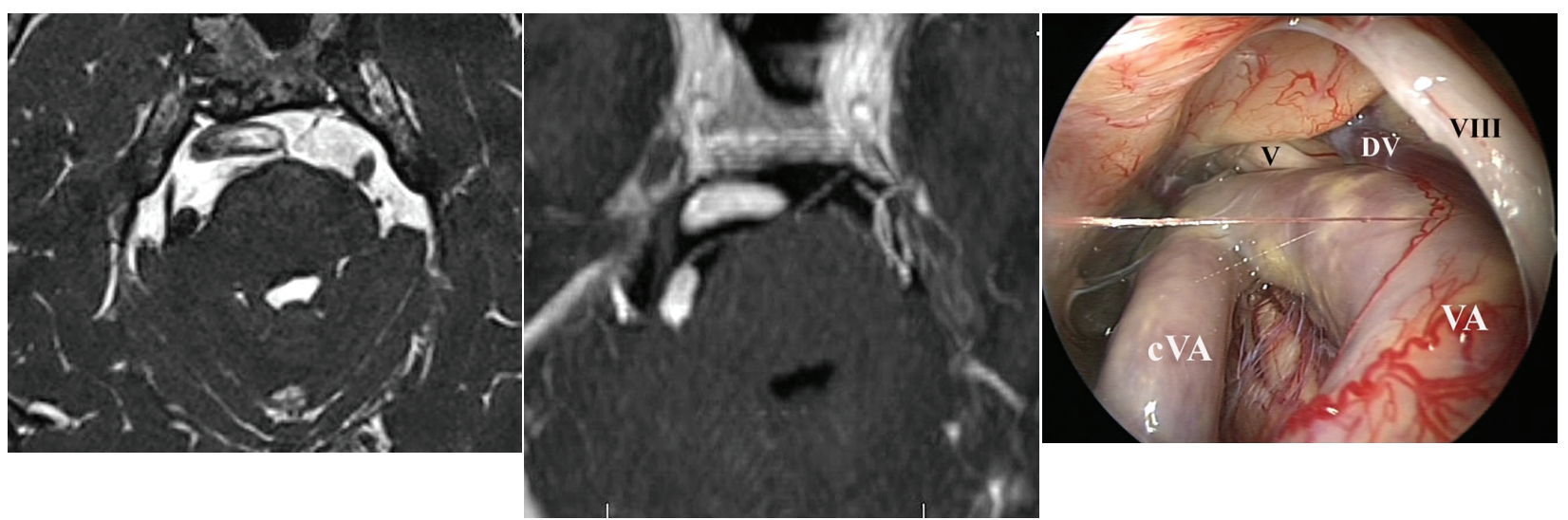

Fig. 4. A. MRI axial T2 CISS sequence. B. T1 gadolinium-enhanced sequence showing an ectasia of the vertebral arteries distorting the right trigeminal nerve. C. Endoscopic view VA (right vertebral artery), cVA (contralateral vertebral artery), $V$ (trigeminal nerve), DV (Dandy vein). 
The second-line drug therapy includes add-on therapy with lamotrigine (400 mg/day) or baclofen (40$80 \mathrm{mg} /$ day) $(13,14)$. As the time passes, however, for many of the patients, the drug become ineffective or the patient develops adverse effects to the drug.

\section{Second Line: Curative}

Surgery is indicated after failure or side effects from the anticonvulsant treatment or when the patient wants a more permanent solution. Endoscopeassisted microvascular decompression may be considered as a treatment of choice providing the only curative solution with a high long-term success rate.

\section{Third Line: Palliative}

Radiofrequency thermocoagulation, glycerol rhizotomy, balloon compression or stereotactic radiotherapy, neuromodulation techniques like Gasserian ganglion stimulation (15), peripheral nerve stimulation (16), and invasive motor cortex stimulation (17), and non-invasive cortex stimulation (18) are considered palliative options. Botulinium neurotoxin type A (BoNT-A) has been experimentally used for the treatment in recent studies (19). Such treatments bypass the cause, the sensory disturbance is constant, the pain recurrence is higher and is not easy to solve by microvascular decompression (MVD) performed after percutaneous ablative techniques or radiotherapy. It can be indicated in the rare case of failure after MVD or in a patient with comorbidity.

\section{pression Surgery}

Endoscope-Assisted Microvascular Decom-

The surgical approach that we follow is keyhole minimally invasive retrosigmoid approach with the patient in the dorsal decubitus position and his head non-fixed, flexed, and turned to the contralateral side.

The retromastoid, retrosigmoid craniotomy is centered on the emissary vein and its size is usually 15 to $20 \mathrm{~mm}$. A "protected" surgical corridor allows to safely access the cranial nerves crossing the cerebellopontine angle. The 30 degrees angled rigid endoscope, $4 \mathrm{~mm}$ in diameter, offers a panoramic view to identify the course of the vascular structure surrounding the trigeminal nerve from the pons medially to the Meckel cave laterally. The offending artery is elevated from the nerve and the pons by dissecting the arachnoid adherences between CN V and the offending vascular loop. The SCA (main trunk or with its two branches) is the most common cause and is

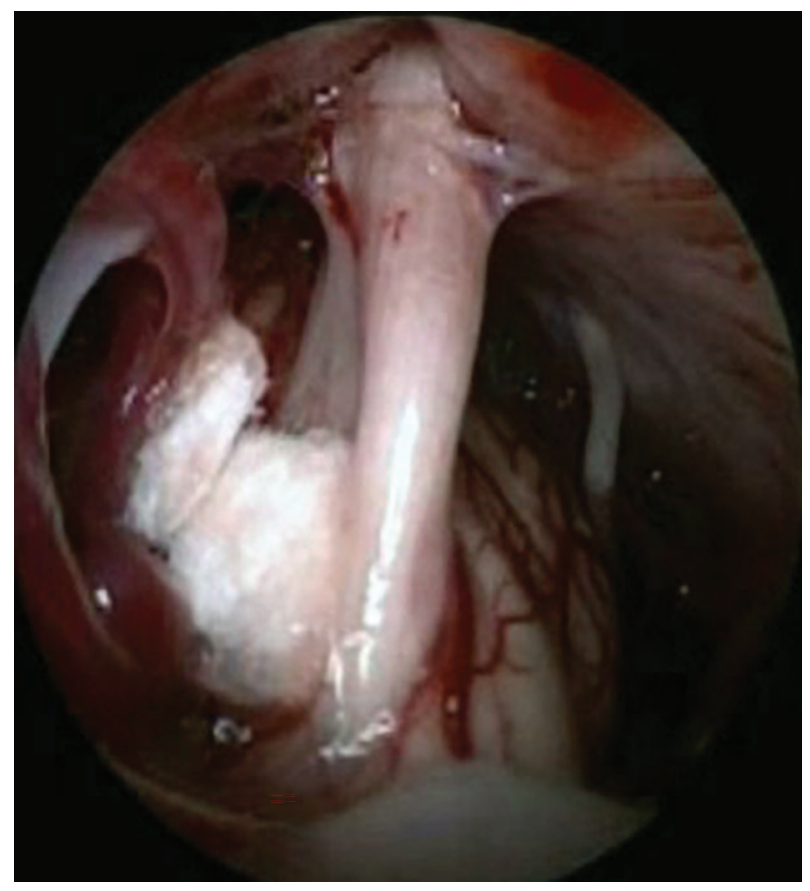

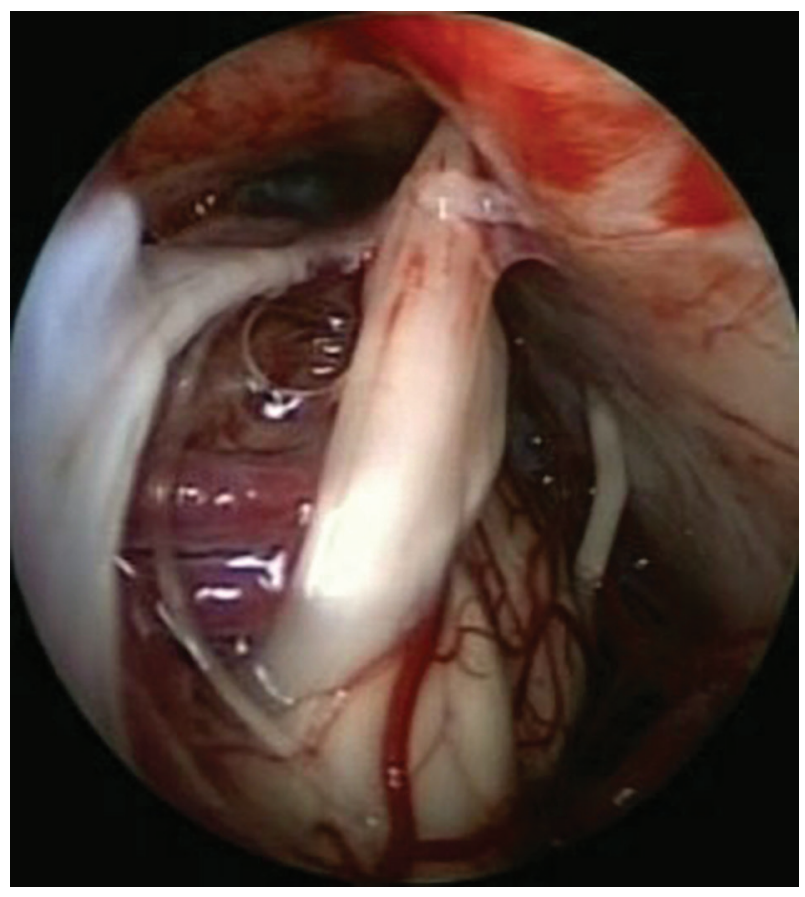

Fig. 5. Endoscope-assisted retrosigmoid approach for a right trigeminal neuralgia: A. The offending structure is a compressive loop of the superior cerebellar artery. B. After decompression of the two branches of SCA away from CN $V$ towards the tentorium with placement of Teflon patches. 
carefully mobilized from the nerve towards the tentorium and maintained far from any contact with the nerve using fibrin glue and small pieces of Teflon (Fig. $5 \mathrm{~A}, \mathrm{~B}$ ). If it is not possible to mobilize the artery further away towards the tentorium, the gap between the offending artery and the nerve should be insulated with a Teflon patch. The vein is rarely mobilized and decompressed with Teflon but mainly coagulated with bipolar coagulation (Fig. 6 A, B). No facial paralysis or hearing loss were present in our series. Post-operative complications were limited to a cerebrospinal fluid (CSF) leak in $2 \%$ of the patients and were solved within one week. A minimally invasive approach as well as its meticulous closure is a key point in preventing complications.

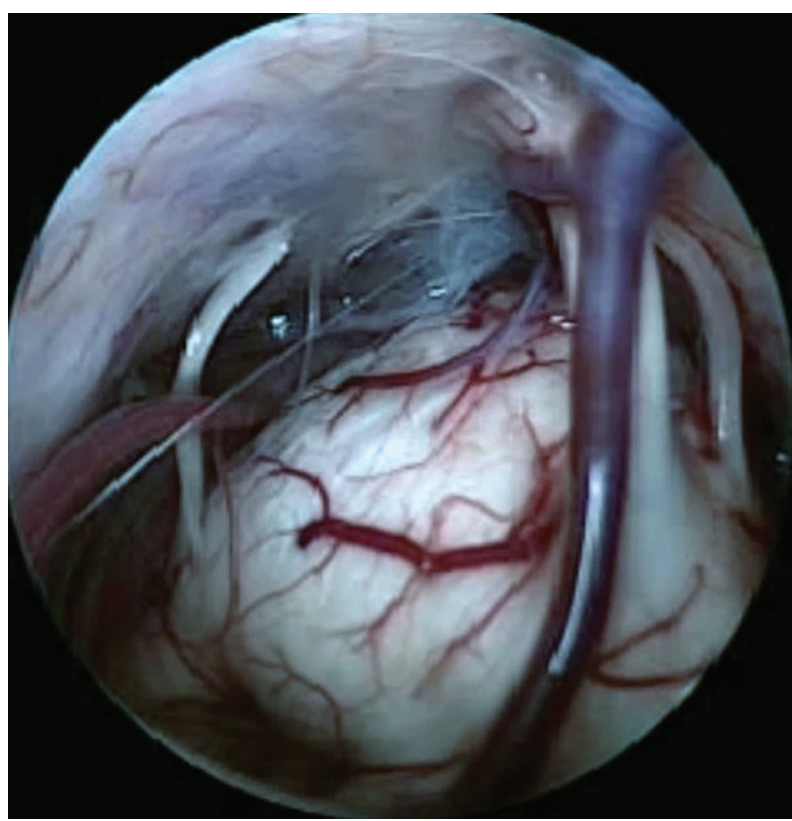

ment. The oldest patient was 87 years old. The complete resolution of the pain was immediate in $88 \%$ and required revision surgery to increase the overall successful result in $93 \%$. The recurrence after immediate postoperative relief is mentioned in all MVD series. The recurrence occurred in $12 \%$ of our patients (26), between 3 months to 2 years after initial surgery. Its main cause is the fibrous tissue around the trigeminal nerve inducing a new neurovascular conflict or embedding the Teflon in the nerve. A revision surgery is recommended and accepted by a patient who has already experienced a pain-free period.

\section{CONCLUSION}

In our neuro-otological experience of more than 1200 retrosigmoid approach surgeries in last

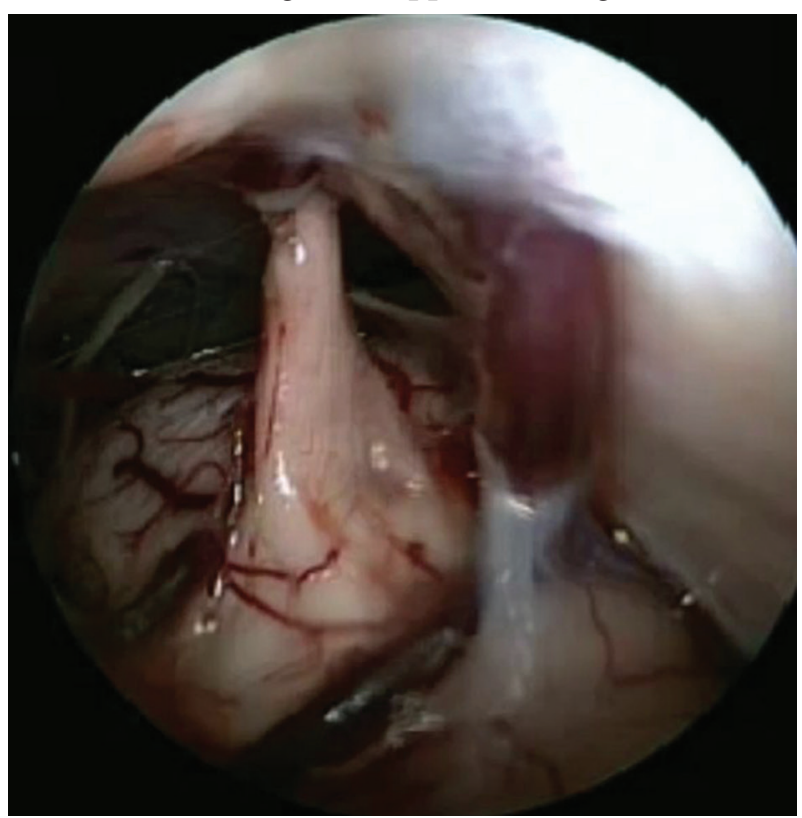

Fig. 6. Endoscope-assisted retrosigmoid approach for a left trigeminal neuralgia: A. Aberrant trigeminal vein crossing inferiorly $C N V$ close to its Meckel cave entrance. B. After coagulation of the offending vein, the trigeminal nerve is free of any vascular contact. The Dandy vein is preserved.

\section{RESULTS OF VASCULAR DECOM- PRESSION FOR TRIGEMINAL NEURALGIA}

In the literature, the success of the procedure varies from $73 \%$ to $97 \%(20-24)$ and confirms that the most sustained pain relief is reported following microvascular decompression (25). We have a combined experience of 355 MVDs on patients suffering from trigeminal neuralgia resistant to medical treat- thirty years for vascular compression syndromes, we have very little morbidity. This should be the rule in the functional surgical indications. The most common surgical cause of incomplete cure is misjudgement of the real offending vessel and incomplete or improper replacement of the conflict vessel. The complementary use of endoscope with the operating microscope enhances the chances of visualization of the offending vessels without increasing the amount of retraction of the cerebellum and thus reducing the 
chances of side effects. Both improvements in anesthesia and in surgery offer a definitive treatment in patients suffering from trigeminal neuralgia at any age.

\section{REFERENCES}

1. Rovit R, Murali R, Jannetta P, editors. Trigeminal neuralgia. Baltimore: Williams \&Wilkins; 1990.

2. Trousseau A. De la nevralgie epileptiforme. Arch Gen Med. 1853; 1:33-44.

3. Fromm G, Terrence C, Maroon J. Trigeminal neuralgia. Current concepts regarding etiology and pathogenesis. Arch Neurol. 1984;41(11):1204-7. doi: 10.1001/archneur.1984.04050220102026.

4. Dandy W. Concerning the cause of trigeminal neuralgia Am J Surg. 1934;24(2):447-55. doi: 10.1016/ S0002-9610(34)90403-7.

5. Gardner J. Concerning the mechanism of trigeminal neuralgia and hemifacial spasm. J Neurosurg. 1962;19:947-58. doi: 10.3171/jns.1962.19.11.0947.

6. Jannetta P. Trigeminal neuralgia and hemifacial spasm- etiology and definitive treatment. Trans Am Neurol Assoc. 1975;100:89-91.

7. Balansard ChF, Meller R, Bruzzo M, Chays A, Girard N, Magnan J. Névralgie du trijumeau: résultats de la décompression vasculaire micro-chirurgicale et endoscopique [Trigeminal neuralgia: results of microsurgical and endoscopic-assisted vascular decompression]. Ann Otolaryngol Chir Cervicofac. 2003;120(6):330-7.

8. Magnan J, Parikh B, Miyazaki H. Functional surgery of cerebellopontine angle by minimally invasive retrosigmoid approach. Jaypee Brothers Medical Publishers; 2013.

9. Elaini S, Miyazaki H, Rameh C, Deveze A, Magnan J. Correlation between magnetic resonance imaging and surgical findings in vasculo-neural compression syndrome. Int Adv Otol. 2009; 5(3):1-23.

10. Tien RD, Wilkins RH. MRA delineation of the vertebro-basilar system in patients with hemifacial spasm and trigeminal neuralgia. AJNR Am J Neuroradiol. 1993; 14(1):34-6.

11. Obermann M. Recent advances in understanding/managing trigeminal neuralgia. F1000Res. 2019;8:F1000 Faculty Rev-505. doi: 10.12688/ f1000research.16092.1.

12. Beydoun A. Safety and efficacy of oxcarbazepine: results of randomized, double-blind trials. Pharma- cotherapy. 2000;20(8 Pt 2):152S-158S. doi: 10.1592/ phco.20.12.152s.35254.

13. Zakrzewska JM, Chaudhry Z, Nurmikko TJ, Patton DW, Mullens LE. Lamotrigine (lamictal) in refractory trigeminal neuralgia: results from a double-blind placebo controlled crossover trial. Pain. 1997;73(2):223-30. doi: 10.1016/ S0304-3959(97)00104-8.

14. Fromm GH, Terrence CF, Chattha AS. Baclofen in the treatment of trigeminal neuralgia: doubleblind study and long-term follow-up. Ann Neurol. 1984;15(3):240-4. doi: 10.1002/ana.410150306.

15. Kustermans L, Van Buyten JP, Smet I, Coucke W, Politis C. Stimulation of the Gasserian ganglion in the treatment of refractory trigeminal neuropathy. J Craniomaxillofac Surg. 2017;45(1):39-46. doi: 10.1016/j.jcms.2016.10.014.

16. Jakobs M, Unterberg A, Treede RD, Schuh-Hofer S, Ahmadi R. Subcutaneous trigeminal nerve field stimulation for refractory trigeminal pain: a cohort analysis. Acta Neurochir (Wien). 2016;158(9):176774. doi: 10.1007/s00701-016-2881-6.

17. Kolodziej MA, Hellwig D, Nimsky C, Benes L. Treatment of central deafferentation and trigeminal neuropathic pain by motor cortex stimulation: Report of a series of 20 patients. J Neurol Surg A Cent Eur Neurosurg. 2016;77(1):52-8. doi: 10.1055/s-0035-1558419.

18. Antal A, Terney D, Kühnl S, Paulus W. Anodal transcranial direct current stimulation of the motor cortex ameliorates chronic pain and reduces short intracortical inhibition. J Pain Symptom Manage. 2010;39(5):890-903. doi: 10.1016/j. jpainsymman.2009.09.023.

19. Aoki KR. Review of a proposed mechanism for the antinociceptive action of botulinum toxin type A. Neurotoxicology. 2005;26(5):785-93. doi: 10.1016/j. neuro.2005.01.017.

20. McLaughlin MR, Jannetta PJ, Clyde BL, Subach BR, Comey CH, Resnick DK. Microvascular decompression of cranial nerves: lessons learned after 4400 operations. J Neurosurg. 1999;90(1):1-8. doi: 10.3171/jns.1999.90.1.0001.

21. El-Garem HF, Badr-El-Dine M, Talaat AM, Magnan J. Endoscopy as a tool in minimally invasive trigeminal neuralgia surgery. Otol Neurotol. 2002;23(2):132-5. doi: 10.1097/00129492-200203000-00004. 
22. Broggi G, Ferroli P, Franzini A, Servello D, Dones I. Microvascular decompression for trigeminal neuralgia: comments on a series of 250 cases, including 10 patients with multiple sclerosis. J Neurol Neurosurg Psychiatry. 2000;68(1):59-64. doi: 10.1136/ jnnp.68.1.59.

23. Sun T, Saito S, Nakai O, Ando T. Long-term results of microvascular decompression for trigeminal neuralgia with reference to probability of recurrence. Acta Neurochir (Wien). 1994;126(2-4):144-8. doi: 10.1007/BF01476425.

24. Oesman C, Mooij JJ. Long-term follow-up of microvascular decompression for trigeminal neuralgia. Skull Base. 2011;21(5):313-22. doi: 10.1055/s-0031-1284213.
25. Cruccu G, Gronseth G, Alksne J, Argoff C, Brainin M, Burchiel K, et al. AAN-EFNS guidelines on trigeminal neuralgia management. Eur J Neurol. 2008;15(10):1013-28. doi: 10.1111/j.1468-1331.2008.02185.x.

26. Zanoletti E, Mazzoni A, Martini A, Abbritti RV, Albertini R, Alexandre E, et al. Surgery of the lateral skull base: a 50-year endeavor. Acta ORL Ital 2019; 39(suppl.1):117-21. doi: 10.14639/0392-100X-suppl.1-39-2019. 